\section{Exploring vascular heterogeneity for gene therapy targeting}

Recent events prompted an increasing debate about the safety and efficacy of gene therapy. ${ }^{1}$ The need to improve available gene therapy vectors to prevent unintended transduction of certain tissues, minimize adverse immune reactions, and promote more efficient expression is clear. ${ }^{2,3}$ Yet the promise of gene therapy is that it is the only treatment specifically designed to correct abnormal gene expression patterns, which are involved in many, if not most, diseases.

The most commonly used gene transfer systems to date originate from viral vectors such as adenovirus, adenoassociated virus, retrovirus, and herpes simplex virus. In theory, some of the concerns over safety and efficacy of these vectors can be addressed by ablating the endogenous nonspecific tropism of the virus and retargeting the vector to a specific site of interest. These modifications may not only prevent an immune response outside the primary target tissue, but also circumvent unintended transduction of other tissues. Furthermore, the efficacy of the vector may be enhanced if the administered dose accumulates in the tissue of interest. Therefore, it is possible that targeted vectors will eventually allow systemic gene therapy with intravenously administered vectors. These advances are of particular significance in multifocal diseases that require systemic treatment, such as metastatic cancer or muscular dystrophy. Although there has been ample research on gene transfer into cancer and non-malignant cells, systemic gene therapy in vivo has been thus far restricted by lack of vector specificity.

Targeting of gene therapy vectors has been achieved mainly in two non-mutually exclusive ways: (1) transcriptional targeting using tissue-specific promoters, and/or (2) receptor targeting by redirecting the receptordependent tropism of the vector. Here, we will focus largely on the latter approach. The use of bispecific conjugates or genetically modified viral capsids and envelopes have modified vector tropism. Bispecific conjugates (containing both a vector-binding and a receptor-binding domain) can, for example, target adenoviral vectors to cells expressing growth factor receptors that are up-regulated during tumor development or inflammation. ${ }^{4-7}$ Ligands for CD3, $\alpha_{V}$ integrins, and heparan sulfate receptors can retarget adenoviruses. ${ }^{8-10}$ Adeno-associated viruses have been targeted by bispecific antibodies to $\alpha_{\text {IIb }} \beta_{3}$ integrins on hematopoietic cells. ${ }^{11}$ Finally, heterologous ligands have been incorporated into the envelopes of retroviruses or the capsids of adenoviruses and adenoassociated viruses; in vitro data suggest that these pro- cedures will enable retargeting these vectors to integrins, $^{12-14} \mathrm{~T}$ cell receptors, ${ }^{15}$ and melanoma-associated antigens. ${ }^{16} \mathrm{~A}$ major limitation of these approaches is that the expression of the targeted receptors is generally not restricted to specific tissues. Specifically in the case of vascular targeted gene therapy, the requirements of selective expression and vector accessibility to cell surface receptors in the blood vessels of the target must be met. $^{17-23}$

To identify ligands that target tissue-specific vascular receptors, we, among others, developed a method that uses phage display peptide libraries (large pools of random peptide sequences displayed on phage particles) administered in vivo. Because tissues modify their endothelium to express tissue-specific vascular receptors, intravenous injection of peptide libraries into mice enables the binding and isolation of homing peptides to specific vascular receptors in different organs or tissues. One can recover and analyze phage bound in the organ of interest in order to identify the peptide sequence mediating the homing. Several organ- and tumor-homing peptides were isolated by this strategy. ${ }^{17-20}$ A unique feature of phage display in vivo applications is that the approach detects the availability of the putative target vascular receptors in the tissue based not only on their expression level, but also on their accessibility to a circulating peptide ligand. Thus, this technology provides novel tools for selective vascular targeting of therapies. ${ }^{20-23}$

Extending these studies, we recently developed a system that uses homing peptides identified by phage display to retarget adenoviral gene therapy vectors. Our approach uses a bispecific adaptor molecule, consisting of two moieties covalently linked: an adenovirus-binding antibody fragment and an organ-homing peptide directed toward a tissue-specific vascular receptor. ${ }^{23}$ As the organ-homing moiety, we have chosen a peptide containing the motif glycine-phenylalanine-glutamic acid (termed GFE). ${ }^{18}$ GFE mediates homing to membrane dipeptidase, a receptor specifically expressed in the lung endothelium. ${ }^{24}$ We used the Fab fragment of the anti-Ad5 neutralizing antibody 1C5IIE11 as the adenovirus-binding moiety. ${ }^{23}$ This recently published work proves the principle that homing peptides can function as part of bispecific adaptors to target gene therapy vectors to vascular receptors specifically expressed in the endothelium of interest.

Gene transfer targeting with bispecific adaptors has potential drawbacks for systemic treatment in vivo, such as instability of the adaptor-vector complex in the blood and additional immunogenicity elicited by the adaptor 
molecule itself. Hence, vascular homing peptides may ultimately require direct integration into the vector's capsid surface. ${ }^{12-16,25}$ However, incorporating homing peptide motifs directly into the viral capsid also has its challenges since peptides may change their three-dimensional conformation when intergrated in the large virus protein which may affect their receptor binding specificity. Currently, phage display is only being used as a tool to isolate homing peptides, but perhaps phage particles could also be used as a targeted gene therapy vector per se. The assumption being that the conformation of the peptide within the phage capsid is optimal for selective vascular homing. As a prokaryotic vector, untargeted (wild-type) phage does not enter eukaryotic cells. When displaying a ligand for a receptor that mediates post-binding internalization, however, phage particles are taken up by mammalian target cells and gene transduction can occur. ${ }^{26}$ Ongoing work in our laboratory suggests that homing phage may be developed as targeted gene therapy vectors (B Restel et al, manuscript in preparation).

In summary, recent work has expanded the possibilities for gene therapy targeting of vascular receptors. Vascular diversity will allow the development of vectors that explore the tissue-specific heterogeneity of blood vessels. The viral homing to receptors in the vasculature after systemic application, perhaps in combination with transcriptional strategies, may restrict nonspecific expression. Tools to achieve these requirements must continue to be developed with the hope that new vectors will lead to targeted gene therapy.

\section{Trepel ${ }^{1,2}, \mathrm{~W}$ Arap $^{1}$ and R Pasqualini ${ }^{1}$ ${ }^{1}$ The University of Texas MD Anderson Cancer Center 1515 Holcombe Boulevard, Box No. 13 Houston, Texas 77030-4095, USA and ${ }^{2}$ Current address: \\ University of Freiburg Medical Center Department of Hematology and Oncology Hugstetter Strasse 55, D-79106 Freiburg, Germany}

\section{References}

1 Marshall E. Gene therapy on trial. Science 2000; 288: 951-957.

2 Verma IM, Somia N. Gene therapy - promises, problems and prospects (news). Nature 1997; 389: 239-242.

3 Vile RG, Russell SJ, Lemoine NR. Cancer gene therapy: hard lessons and new courses. Gene Therapy 2000; 7: 2-8.

4 Douglas JT et al. Targeted gene delivery by tropism-modified adenoviral vectors. Nat Biotechnol 1996; 14: 1574-1578.

5 Goldman CK et al. Targeted gene delivery to Kaposi's sarcoma cells via the fibroblast growth factor receptor. Cancer Res 1997; 57: 1447-1451.

6 Watkins SJ et al. The 'adenobody' approach to viral targeting: specific and enhanced adenoviral gene delivery. Gene Therapy 1997; 4: 1004-1012.

7 Miller CR et al. Differential susceptibility of primary and established human glioma cells to adenovirus infection: targeting via the epidermal growth factor receptor achieves fiber receptorindependent gene transfer. Cancer Res 1998; 58: 5738-5748.

8 Wickham TJ et al. Targeted adenovirus gene transfer to endothelial and smooth muscle cells by using bispecific antibodies. J Virol 1996; 70: 6831-6838.

9 Wickham TJ et al. Adenovirus targeted to heparan-containing receptors increases its gene delivery efficiency to multiple cell types. Nat Biotechnol 1996; 14: 1570-1573.

10 Wickham TJ et al. Targeted adenovirus-mediated gene delivery to T cells via CD3. J Virol 1997; 71: 7663-7669.

11 Bartlett JS et al. Targeted adeno-associated virus vector transduction of nonpermissive cells mediated by a bispecific Fab'gamma) ${ }_{2}$ antibody. Nat Biotechnol 1999; 17: 181-186.

12 Dmitriev I et al. An adenovirus vector with genetically modified fibers demonstrates expanded tropism via utilization of a coxsackievirus and adenovirus receptor-independent cell entry mechanism. J Virol 1998; 72: 9706-9713.

13 Vigne $\mathrm{E}$ et al. RGD inclusion in the hexon monomer provides adenovirus type 5-based vectors with a fiber knob-independent pathway for infection. J Virol 1999; 73: 5156-5161.

14 Girod A et al. Genetic capsid modifications allow efficient retargeting of adeno-associated virus type 2. Nat Med 1999; 5: 1052-1056

15 Engelstadter $\mathrm{M}$ et al. Targeting human $\mathrm{T}$ cells by retroviral vectors displaying antibody domains selected from a phage display library. Hum Gene Ther 2000; 11: 293-303.

16 Martin $\mathrm{F}$ et al. Retrovirus targeting by tropism restriction to melanoma cells. J Virol 1999; 73: 6923-6929.

17 Pasqualini R, Ruoslahti E. Organ targeting in vivo using phage display peptide libraries. Nature 1996; 380: 364-366.

18 Rajotte D et al. Molecular heterogeneity of the vascular endothelium revealed by in vivo phage display. J Clin Invest 1998; 102: 430-437.

19 Pasqualini R, Koivunen E, Ruoslahti E. Alpha v integrins as receptors for tumor targeting by circulating ligands. Nat Biotechnol 1997; 15: 542-546.

20 Arap W, Pasqualini R, Ruoslahti E. Cancer treatment by targeted drug delivery to tumor vasculature in a mouse model. Science 1998; 279: 377-380.

21 Ellerby HM et al. Anti-cancer activity of targeted pro-apoptotic peptides. Nat Med 1999; 5: 1032-1038.

22 Koivunen $\mathrm{E}$ et al. Tumor targeting with a selective gelatinase inhibitor. Nat Biotechnol 1999; 17: 768-774.

23 Trepel $\mathrm{M}$ et al. Molecular adaptors for vascular-targeted adenoviral gene delivery. Hum Gene Ther 2000; 11: 1971-1981.

24 Rajotte D, Ruoslahti E. Membrane dipeptidase is the receptor for a lung-targeting peptide identified by in vivo phage display. J Biol Chem 1999; 274: 11593-11598.

25 Wickham TJ, Carrion ME, Kovesdi I. Targeting of adenovirus penton base to new receptors through replacement of its RGD motif with other receptor-specific peptide motifs. Gene Therapy 1995; 2: 750-756.

26 Larocca $\mathrm{D}$ et al. Gene transfer to mammalian cells using genetically targeted filamentous bacteriophage. FASEB J 1999; 13: 727-734 